\title{
Significant Enhancement of Piezoelectric Response in AlN by Yb Addition
}

\author{
Kenji Hirata ${ }^{1, *(\mathbb{D}}$, Yuto Mori ${ }^{2}$, Hiroshi Yamada ${ }^{1,2}$, Masato Uehara ${ }^{1,2}{ }^{-}$, Sri Ayu Anggraini ${ }^{1}$ and Morito Akiyama ${ }^{1}$ \\ 1 Sensing System Research Center, National Institute of Advanced Industrial Science and Technology (AIST), \\ Shuku 807-1, Tosu, Saga 841-0052, Japan; hiro-yamada@aist.go.jp (H.Y.); m.uehara@aist.go.jp (M.U.); \\ ayu-anggraini@aist.go.jp (S.A.A.); m.akiyama@aist.go.jp (M.A.) \\ 2 Department of Molecular and Material Sciences, Interdisciplinary Graduate School of Engineering Sciences, \\ Kyushu University, 6-1 Kasugakoen, Kasuga, Fukuoka 816-8580, Japan; mori.yuto.nagasaki@gmail.com \\ * Correspondence: kenji.hirata@aist.go.jp; Tel.: +81-942-81-3658
}

check for updates

Citation: Hirata, K.; Mori, Y.;

Yamada, H.; Uehara, M.; Anggraini,

S.A.; Akiyama, M. Significant Enhancement of Piezoelectric Response in AlN by $\mathrm{Yb}$ Addition. Materials 2021, 14, 309. https:// doi.org/10.3390/ma14020309

Received: 18 December 2020 Accepted: 7 January 2021 Published: 9 January 2021

Publisher's Note: MDPI stays neutral with regard to jurisdictional clai$\mathrm{ms}$ in published maps and institutional affiliations.

Copyright: (C) 2021 by the authors. Licensee MDPI, Basel, Switzerland. This article is an open access article distributed under the terms and conditions of the Creative Commons Attribution (CC BY) license (https:// creativecommons.org/licenses/by/ $4.0 /)$.

\begin{abstract}
This study employs first-principles calculations to investigate how introducing $\mathrm{Yb}$ into aluminum nitride (AlN) leads to a large enhancement in the material's piezoelectric response $\left(d_{33}\right)$. The maximum $d_{33}$ is calculated to be over $100 \mathrm{pC} / \mathrm{N}$, which is 20 times higher than that of AlN. One reason for such a significant improvement in $d_{33}$ is the elastic-softening effect, which is indicated by a decrease in the elastic constant, $C_{33}$. The strain sensitivity $(d u / d \varepsilon)$ of the internal parameter, $u$, is also an important factor for improving the piezoelectric stress constant, $e_{33}$. On the basis of mixing enthalpy calculations, $\mathrm{Yb}_{x} \mathrm{Al}_{1-x} \mathrm{~N}$ is predicted to be more stable as a wurtzite phase than as a rock salt phase at composition up to $x \approx 0.7$. These results suggest that $\mathrm{Yb}$ can be doped into $\mathrm{AlN}$ at high concentrations. It was also observed that the dielectric constant, $\varepsilon_{33}$, generally increases with increasing $\mathrm{Yb}$ concentrations. However, the electromechanical coupling coefficient, $k_{33}^{2}$, only increases up to $x=0.778$, which is likely because of the relatively lower values of $\varepsilon_{33}$ within this range.
\end{abstract}

Keywords: first-principles calculation; piezoelectric material; nitride; thin film

\section{Introduction}

The piezoelectric properties of aluminum nitride (AlN)-based wurtzite solid solutions have been investigated experimentally and theoretically [1-11]. In particular, scandium (Sc)-doped AlN (ScAlN) exhibits high piezoelectricity and has been widely considered for use in high-frequency filters, sensors, and microelectromechanical devices [12,13]. Ferroelectricity has also been observed with ScAlN, so its scope of potential applications is expected to expand [14].

Recently, an enhanced piezoelectric response was discovered in ytterbium (Yb)-doped AlN (YbAlN) [15]. However, there are very few systematic experimental reports on the phase stability and piezoelectric properties of wurtzite phase YbAlN. Although both $\mathrm{Yb}$ and Sc are classified as rare earth elements, it is still unclear whether the piezoelectric properties of $\mathrm{YbAlN}$ are equivalent to those of ScAlN. Since the piezoelectric properties of $\mathrm{YbAlN}$ have never been investigated in detail, it is unknown whether the observed improvement in piezoelectric response occurs via the same mechanism as previously reported AlN-based piezoelectric materials. The piezoelectric constant, $d_{33}$, can be approximated as $d_{33} \approx e_{33} / C_{33}$, where $e_{33}$ is the piezoelectric stress constant and $C_{33}$ is the elastic constant. Computational results have indicated that elastic softening (decrease of $C_{33}$ ) can be caused by the addition of other elements into $\operatorname{AlN}[4,7,9,11]$. Furthermore, the lattice parameter ratio $(c / a)$ of the wurtzite structure decreases as the quantity of additional elements increases $[1,2,9,16]$. Changes in the piezoelectric stress constant, $e_{33}$, are generally corelated with changes in the lattice parameter [17].

Typically, AlN-based piezoelectric materials are fabricated as thin films using a sputtering technique, and the solubility of the additive elements depends heavily on the sputtering conditions. The stability of the wurtzite phase is an important factor for designing 
AlN-based piezoelectric materials. The phase stability of rock salt (which is thermodynamically competitive with the wurtzite phase) has been evaluated by first-principles calculations $[6,7,11,16]$, and the mixing enthalpies of the wurtzite and rock salt phases have been calculated, allowing for estimations of the stable composition of each phase $[6,11]$. The experimental results were generally consistent with the calculated results $[1,6,11]$, so evaluations based on mixing enthalpy are considered reasonable. However, the thermodynamic stability of the wurtzite phase should also be evaluated for YbAlN.

In the present work, $\mathrm{YbAlN}$ is evaluated in terms of the lattice constant of its crystal structure, its phase stability, and the piezoelectric properties of its wurtzite phase using first-principles calculations.

\section{Computational Methods}

The atomic configuration of YbAlN was constructed using a Special Quasirandom Structures method [18], which was implemented in Alloy Theoretic Automated Toolkit [19]. The sizes of the supercells were $3 \times 3 \times 2$ (72 atoms), $2 \times 2 \times 2$ (64 atoms), and $3 \times 3 \times 2$ (72 atoms) for wurtzite, rock salt, and non-polar hexagonal phases, respectively. The crystal structure of each phase is shown in Figure $1 . \mathrm{Yb}$ atoms substituted $\mathrm{Al}$ sites in each supercell model. For structural optimization, we used the Vienna Ab initio Simulation Package (VASP) based on density functional theory $[20,21]$. The exchange and correlation functions were given by the generalized gradient approximation, as proposed by Perdew et al. [22]. The Blöchl's projector-augmented wave (PAW) method was implemented as described by Kresse and Joubert [23,24]. The Monkhorst-Pack method [25] was employed for $k$-point sampling, and the meshes of the wurtzite, rock salt, and non-polar hexagonal phases were $3 \times 3 \times 2,2 \times 2 \times 3$, and $3 \times 3 \times 2$, respectively. A cutoff energy of $500 \mathrm{eV}$ was used for the plane-wave expansion. Furthermore, an on-site coulomb interaction was introduced for the ytterbium f-state. The strong correlation between localized $\mathrm{d}$ electrons was described using the parameter, $U$, which is defined as $U=U^{\text {sic }}-J$, where parameter $U^{s i c}$ is the on-site coulomb self-interaction correction potential and $J$ is the exchange integral. In this work, we used $U^{\text {sic }}=10.329 \mathrm{eV}$ and $J=1.349 \mathrm{eV}[26]$.

(a)

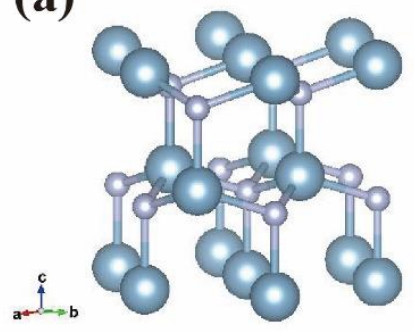

(b)

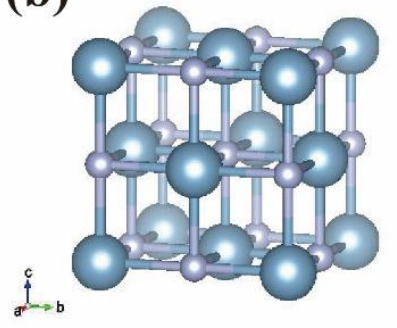

(c)

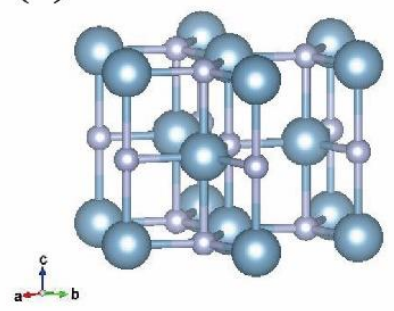

Figure 1. The crystal structures of (a) wurtzite, (b) rock salt, and (c) non-polar hexagonal phases. The blue and gray spheres represent the metal and nitrogen atoms, respectively.

To calculate the mixing enthalpy, we performed a total energy calculation after structural optimization using the tetrahedron method with Blöchl corrections. The mixing enthalpy of nitride is described in Equation (1),

$$
\Delta H_{\text {mix }}=H\left(\mathrm{Yb}_{x} \mathrm{Al}_{1-x} N\right)-x H_{Y b N}^{\text {rock salt }}-(1-x) H_{A l N}^{\text {wurtzite }}
$$

where $H\left(\mathrm{Yb}_{x} \mathrm{Al}_{1-x} N\right)$ is the total energy of each phase, and $H_{Y b N}^{\text {rock salt }}$ and $H_{A l N}^{\text {wurtzite }}$ are the total energy of $\mathrm{YbN}$ (with rock salt structure) and AlN (with wurtzite structure), respectively.

Phonon and dielectric response calculations based on finite differences and density functional perturbation theory [27-29] were performed to calculate the elastic ten- 
sors and piezoelectric stress tensors. The piezoelectric constant, $d_{33}$, is determined using Equation (2),

$$
d_{33}=\frac{e_{33}\left(C_{11}+C_{12}\right)-2 e_{31} C_{13}}{\left(C_{11}+C_{12}\right) C_{33}-2 C_{13}^{2}}
$$

where $e_{\mathrm{ij}}$ and $C_{\mathrm{ij}}$ are the piezoelectric stress constant and elastic constant, respectively.

\section{Results and Discussion}

\subsection{Evaluation of Mixing Enthalpies}

The formation of the $\mathrm{YbN}$ rock salt phase has been observed in the $\mathrm{Yb}-\mathrm{N}$ binary system [30], and the thermodynamic stabilities of the wurtzite and rock salt phases are believed to compete in an AlN-YbN pseudo-binary system. The calculated mixing enthalpies of wurtzite, rock salt, and non-polar hexagonal phases are shown in Figure 2. The wurtzite phase has a smaller mixing enthalpy than the rock salt phase, and it is thermodynamically stable up to $x=0.7$. The calculated mixing enthalpy indicates that the rock salt phase becomes more stable with the higher concentration of $\mathrm{Yb}(x>0.7)$. To our knowledge, the phase stability of an AlN-YbN pseudo-binary system has not yet been evaluated experimentally; however, the lattice constant of rock salt $\mathrm{YbN}$ has been estimated as $a=4.79 \mathrm{~nm}$ [30], which is consistent with our calculated lattice constant, $a=4.78 \mathrm{~nm}$. Generally, for thin films of AlN-based piezoelectric materials fabricated by the sputtering method, the stable phase region is estimated near the cross point of the mixing enthalpies of the rock salt and wurtzite phases $[6,11]$. On the basis of this concept, the wurtzite phase can potentially be obtained in a thin film until around $x=0.6$ in $\mathrm{Yb}_{x} \mathrm{Al}_{1-x} \mathrm{~N}$. This tendency is similar to ScAlN [1] and MgNbAlN [2], which are reported to have a wide solubility in the wurtzite phase.

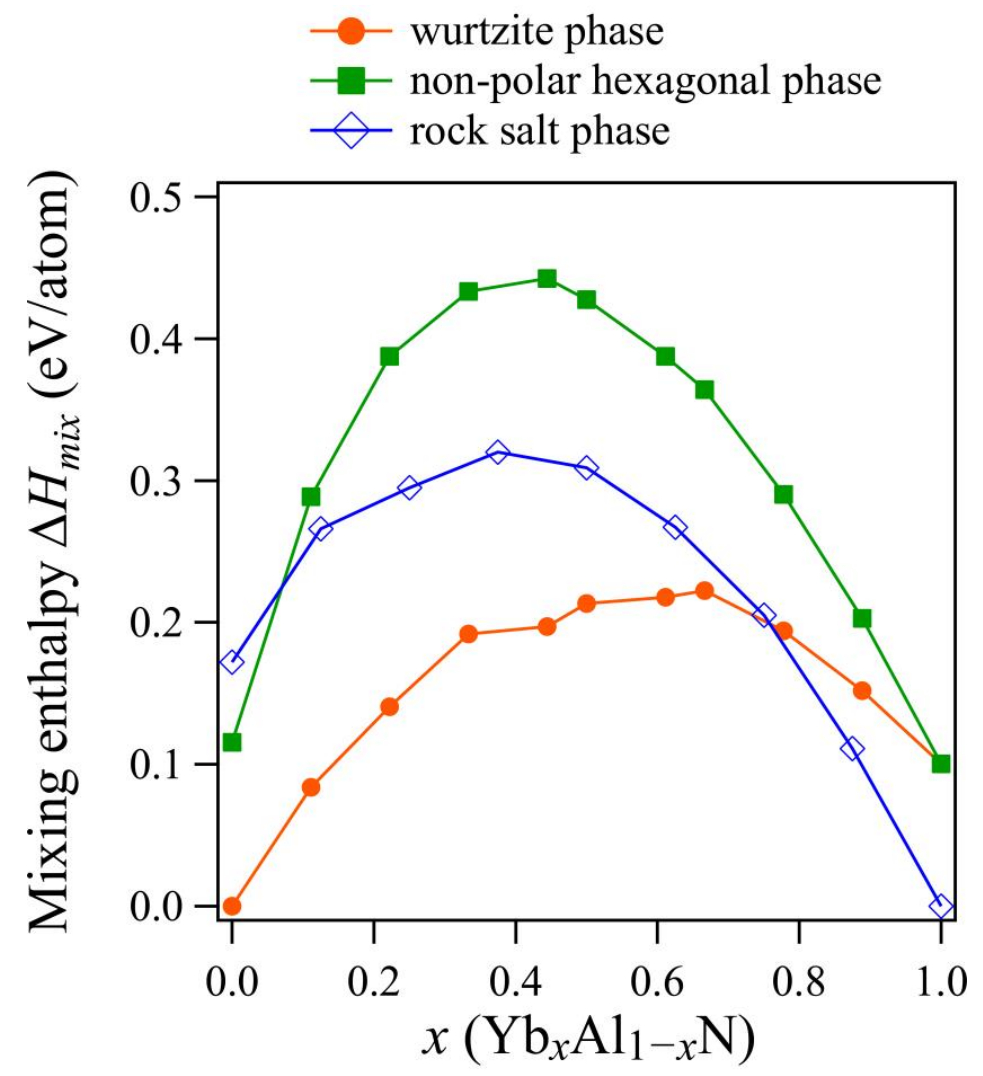

Figure 2. Calculated mixing enthalpies of each phase in $\mathrm{Yb}_{x} \mathrm{Al}_{1-x} \mathrm{~N}$. Blue, orange, and green traces correspond to the mixing enthalpies of rock salt, wurtzite, and non-polar hexagonal phases, respectively. 
To evaluate the structural stability of the wurtzite phase, the mechanical stability was also investigated. The mechanical stability criteria for hexagonal symmetry [31] are evaluated by using the elastic stiffness constants $C_{\mathrm{ij}}$, which are described as following inequality.

$$
C_{44}>0, C_{11}>\left|C_{12}\right|,\left(C_{11}+2 C_{12}\right) C_{33}>2 C_{13}^{2}
$$

The elastic stiffness constants are listed in Table 1 , which were used for inequality (3). In all compositions, the elastic constants of $\mathrm{Yb}_{x} \mathrm{Al}_{1-x} \mathrm{~N}$ satisfy the mechanical stability criteria, and the structural stability of the compounds is considered to be ensured.

Table 1. Elastic stiffness constants of $\mathrm{Yb}_{x} \mathrm{Al}_{1-x} \mathrm{~N}$.

\begin{tabular}{cccccc}
\hline \multirow{2}{*}{ Concentration: $\boldsymbol{x}$} & \multicolumn{5}{c}{ Elastic Stiffness Constant (GPa) } \\
\cline { 2 - 5 } & $\boldsymbol{C}_{\mathbf{1 1}}$ & $\boldsymbol{C}_{\mathbf{1 2}}$ & $\boldsymbol{C}_{\mathbf{1 3}}$ & $\boldsymbol{C}_{\mathbf{3 3}}$ & $\boldsymbol{C}_{\mathbf{4 4}}$ \\
\hline 0.000 & 377.6 & 128.4 & 97.9 & 358.5 & 124.3 \\
0.111 & 324.1 & 131.4 & 111.7 & 288.0 & 92.2 \\
0.222 & 289.0 & 129.3 & 110.6 & 243.3 & 77.0 \\
0.333 & 256.5 & 122.0 & 116.7 & 214.8 & 68.5 \\
0.444 & 233.7 & 134.3 & 120.6 & 173.1 & 49.3 \\
0.500 & 231.5 & 134.2 & 110.5 & 146.1 & 41.7 \\
0.611 & 206.7 & 120.6 & 117.9 & 136.0 & 33.5 \\
0.667 & 199.5 & 131.9 & 118.4 & 116.7 & 31.5 \\
0.778 & 202.3 & 140.8 & 101.3 & 165.2 & 30.1 \\
0.889 & 201.3 & 149.5 & 89.0 & 233.7 & 24.1 \\
1.000 & 197.9 & 151.7 & 72.5 & 321.1 & 23.2 \\
\hline
\end{tabular}

The difference of mixing enthalpy between the wurtzite and the non-polar hexagonal phases tends to increase in the range of $0 \leq x \leq 0.5$, and it decrease with increasing in the concentration range of $0.5<x \leq 1.0$. It has been suggested that differences in mixing enthalpy affect the coordination of metal atoms in the wurtzite structure, which can lead to a drastic decrease in the lattice constant ratio, $c / a$. Therefore, it is important to discuss the relationship between the differences in mixing enthalpies and the changes in the lattice constants of the wurtzite phase in YbAlN.

\subsection{Evaluation of Lattice Parameters}

Figure 3 shows the calculated lattice parameters of $\mathrm{Yb}_{x} \mathrm{Al}_{1-x} \mathrm{~N}$. The lattice constants of AlN were calculated as $a=3.13 \mathrm{~nm}$ and $c=5.02 \mathrm{~nm}$; these values are consistent with the experimental values $a=3.11 \mathrm{~nm}$ and $c=4.98 \mathrm{~nm}$ reported by Wang et al. [32]. The lattice constant, $a$, increased monotonically as the $\mathrm{Yb}$ concentration increased. In contrast, the lattice constant, $c$, elongated slightly until the $\mathrm{Yb}$ concentration reached $x=0.333$ and then shortened gradually as the $\mathrm{Yb}$ concentration increased further. Additionally, $c / a$ decreased to $\approx 1.2$ around a $\mathrm{Yb}$ concentration of $x=1.0$, and the atomic configuration shifted toward a non-polar hexagonal structure. Similar behavior has been observed in other AlN-based piezoelectric materials [11,16]. Moreover, the mixing enthalpy of the non-polar hexagonal phase is close to that of the wurtzite phase at around $x=1.0$ (see Figure 2). In the composition where $x=1.0$, the $c / a$ of the wurtzite phase is estimated around to be 1.2, which is almost equal to that of the non-polar hexagonal phase. This composition corresponds to a neighboring point between the non-polar hexagonal and wurtzite phases, which is consistent with calculations regarding ScAlN [11]. However, the composition of the neighboring point shifts to higher $x$ values in YbAlN than in ScAlN $(x=0.8)[11]$. 


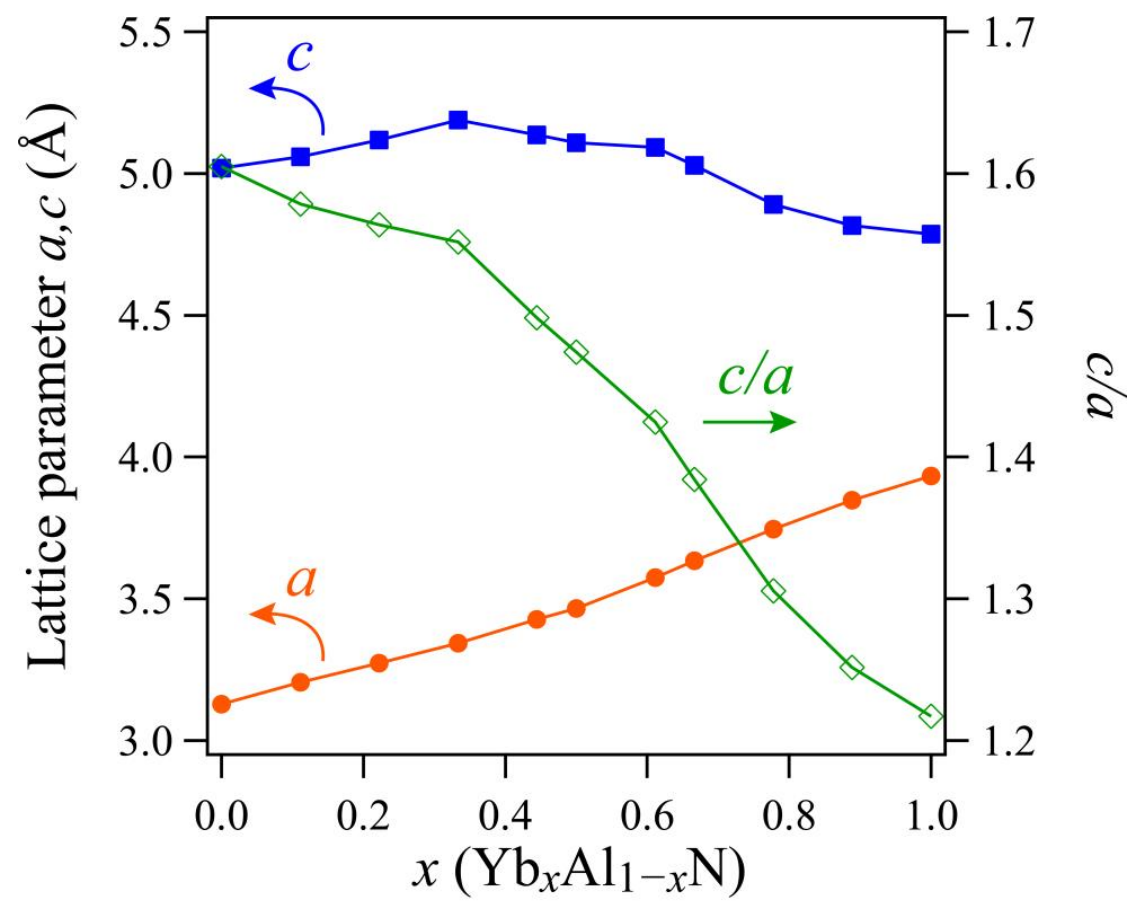

Figure 3. Calculated lattice parameters of $\mathrm{Yb}_{x} \mathrm{Al}_{1-x} \mathrm{~N}$. Orange and blue traces represent the changes to lattice parameters, $a$ and $c$, respectively, and the green line denotes the ratio of lattice parameters, $c / a$.

\subsection{Evaluation of Piezoelectric Constants}

Figure 4a presents the calculated piezoelectric stress constant, $e_{33}$, and elastic constant, $C_{33}$, and it shows that $e_{33}$ increases and $C_{33}$ decreases with increasing concentrations of $\mathrm{Yb}$. The piezoelectric constant, $d_{33}$, can be approximated by $d_{33} \approx e_{33} / C_{33}$, so increasing $e_{33}$ and decreasing $C_{33}$ lead to an increase in $d_{33}$, as shown in Figure $4 \mathrm{~b}$. This phenomenon has also been reported for other metal-doped AlN [11]. The maximum $d_{33}$ value was estimated to be over $100 \mathrm{pC} / \mathrm{N}$, which is almost equal to the maximum theoretical $d_{33}$ of ScAlN [11] and about 20 times that of AlN.
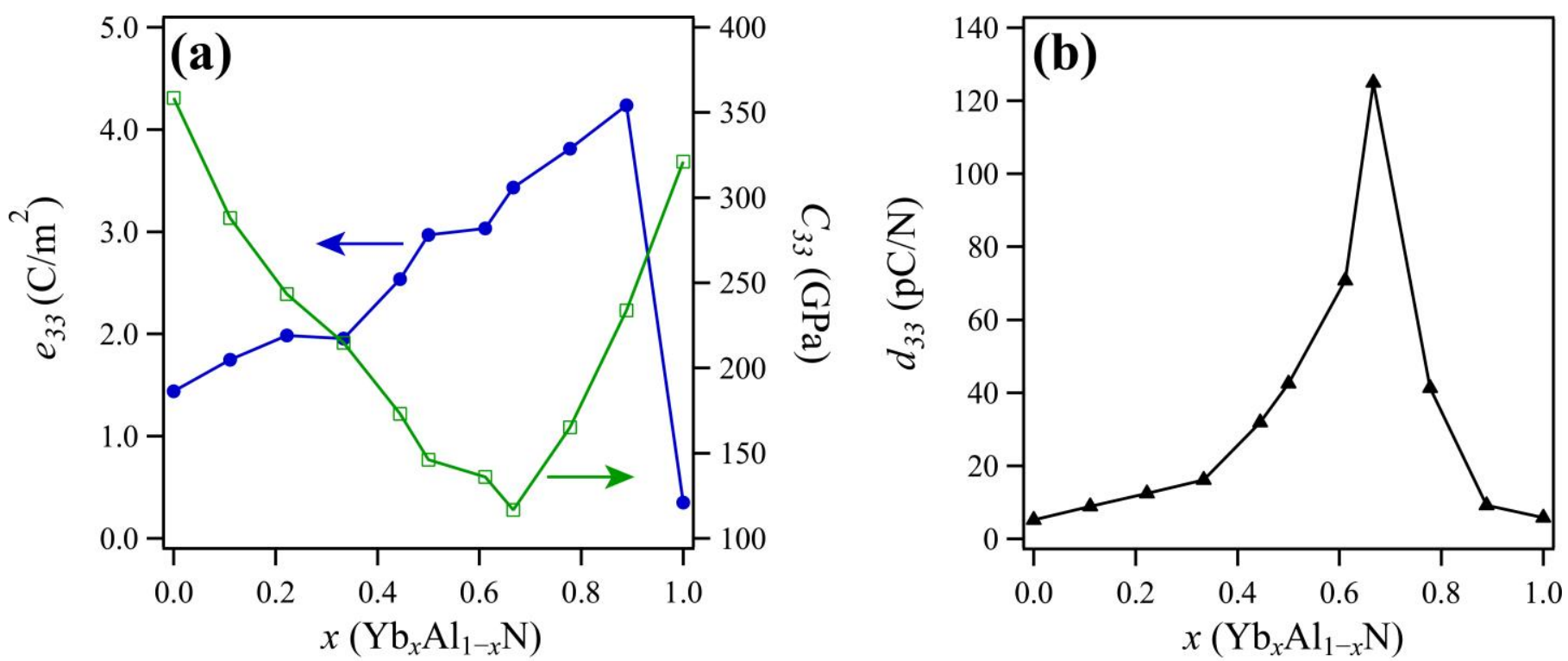

Figure 4. (a) Calculated piezoelectric stress constant, $e_{33}$ (blue line), and elastic constant, $C_{33}$ (green line) of $\mathrm{Yb}_{x} \mathrm{Al}_{1-x} \mathrm{~N}$ as a function of $\mathrm{Yb}$ content; (b) calculated piezoelectric constant, $d_{33}$, of $\mathrm{Yb}_{x} \mathrm{Al}_{1-x} \mathrm{~N}$ as a function of $\mathrm{Yb}$ content. 
To understand why the addition of $\mathrm{Yb}$ can enhance the piezoelectric stress constant, $e_{33}$, we evaluated each parameter comprising $e_{33}$, as shown in Equation (4),

$$
e_{33}=e_{33}^{\text {clamped }}+e_{33}^{\text {nonclamped }}
$$

where $e_{33}^{\text {clamped }}$ is the electronic response to strain (evaluated in the equilibrium positions with fixed internal atomic coordinates). The $e_{33}^{\text {nonclamped }}$ term is the contribution from the internal atomic coordinates in response to an external strain along the $c$-direction, and this term can be defined as shown in Equation (5),

$$
e_{33}^{\text {nonclamped }}=\frac{4 e Z_{33}}{\sqrt{ } 3 a^{2}} \frac{d u}{d \varepsilon}
$$

where $e$ is the (positive) electron charge, $a$ is the equilibrium lattice constant, $Z_{33}$ is the Born effective charge (in units of $e$ ), and $\varepsilon$ is the macroscopic applied strain. The internal parameter, $u$, represents the ratio between the lattice constant, $c$, and the metal-nitrogen distance along the $c$-axis in the wurtzite structure (see Figure $5 \mathrm{a}$ ). The calculated results for these terms are shown in Figure $5 \mathrm{~b}-\mathrm{e}$. The $e_{33}^{\text {clamped }}$ value decreased until the concentration of $\mathrm{Yb}$ reached $x=0.333$, and it did not seem to contribute to the observed increase of $e_{33}$ in this range of composition; however, at $\mathrm{Yb}$ concentrations higher than $x=0.333$, $e_{33}^{\text {clamped }}$ increased and did contribute to the improvement of $e_{33}$. The $e_{33}^{\text {nonclamped }}$ increased up to a $\mathrm{Yb}$ concentration $x=0.889$, resulting in a value that was about twice that of AlN. Considering its magnitude, $e_{33}^{\text {nonclamped }}$ had a greater effect on the enhancement of $e_{33}$ than $e_{33}^{\text {clamped }}$. Similar trends have been observed in $\mathrm{ScAlN}$, so it is reasonable to believe that the displacement of ions contributes more to the enhancement of $e_{33}$ than the displacement of electrons [4]. It is clear from Figure $5 \mathrm{~d}$ that $Z_{33}$ increased monotonically as the $\mathrm{Yb}$ concentration increased, and it reached about 1.5 times the $Z_{33}$ value of AlN. Similarly, the strain sensitivity of the internal parameter $(d u / d \varepsilon)$ increased as the $\mathrm{Yb}$ concentration increased. Specifically, as the $\mathrm{Yb}$ concentration increased from $x=0$ to $x=0.889, d u / d \varepsilon$ increased 2.5 times, while $Z_{33}$ only increased 1.5 times, indicating that the $d u / d \varepsilon$ parameter has a stronger influence in terms of increasing $e_{33}^{\text {nonclamped }}$. A large $d u / d \varepsilon$ value means that the displacement of atoms in the alloy is large when it experiences external strain; therefore, this factor contributes significantly to the enhancement of $e_{33}$. The maximum value of $d u / d \varepsilon$ was obtained around the composition where the $c / a$ of the wurtzite phase and the non-polar hexagonal phase are the same. Similar behavior was observed in ScAlN [11], where the composition with the smallest difference between wurtzite and non-polar hexagonal phase mixing enthalpies (almost zero) also had the maximum $e_{33}$. The piezoelectric properties of YbAlN are believed to be enhanced through a similar mechanism as for ScAlN. Since the lowest $C_{33}$ value and the highest $d u / d \varepsilon$ value occur at the almost same $\mathrm{Yb}$ concentration, the increase in $d u / d \varepsilon$ may be related to the elastic softening. 

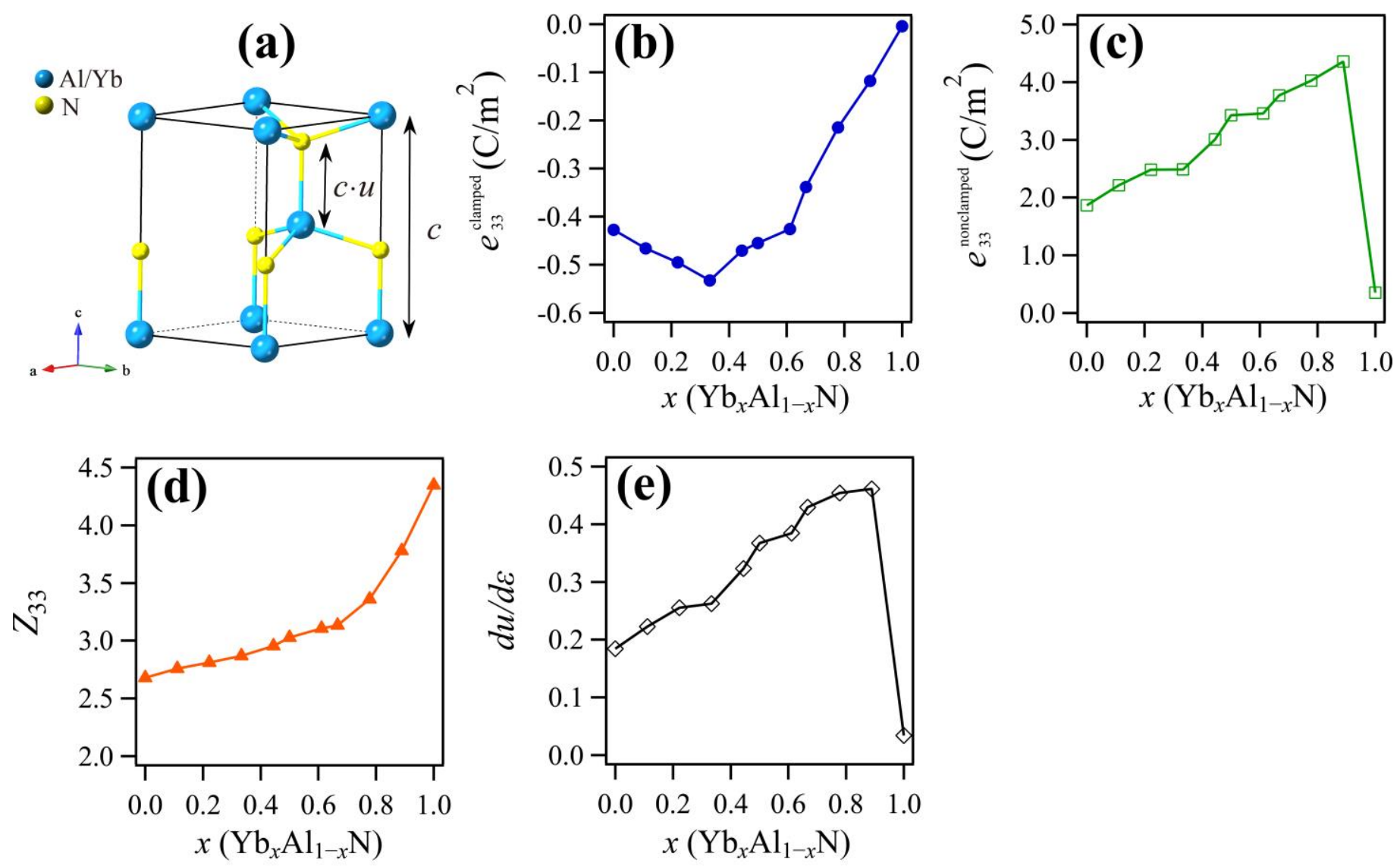

Figure 5. (a) Crystal structure of wurtzite showing the internal parameter, $u$. Blue and yellow spheres represent $\mathrm{Al} / \mathrm{Yb}$ and $\mathrm{N}$ atoms, respectively. Other changes to the component values of $e_{33}$ as a function of $\mathrm{Yb}$ content; (b) clamped $e_{33}$; (c) nonclamped $e_{33} ;$ (d) components of the nonclamped Born effective charge, $Z_{33}$; and (e) the strain sensitivity of the internal parameter, $d u / d \varepsilon$.

\subsection{Evaluation of the Electromechanical Coupling Constant}

To verify the reliability of calculated piezoelectric properties, it is necessary to compare the calculated values with the experimental values. There is limited experimental data available regarding the piezoelectric properties of $\mathrm{YbAlN}$; however, the electromechanical coupling constants $\left(k_{33}^{2}\right)$ reported by Yanagitani et al. [15] can be used to confirm the reliability of our calculated results. The $k_{33}^{2}$ values were calculated using Equation (6),

$$
k_{33}^{2}=\frac{e_{33}^{2}}{\varepsilon_{33} C_{33}+e_{33}^{2}}
$$

where $\varepsilon_{33}$ is the 33 components of the dielectric tensor, which are plotted in Figure $6 a$. Figure $6 \mathrm{~b}$ shows the $k_{33}^{2}$ values calculated in this work, as well as the experimental and calculated values reported by Yanagitani et al. [15]. For pure AlN, $\varepsilon_{33}$ is calculated as 9.77, and this value is consistent with the previous computational result $\left(\varepsilon_{33}=9.74\right)[33,34]$. The value of $\varepsilon_{33}$ increased monotonically as the $\mathrm{Yb}$ concentration in $\mathrm{Yb}_{x} \mathrm{Al}_{1-x} \mathrm{~N}$ increased. Similarly, $k_{33}^{2}$ increased until $x=0.778$, and then, it decreased as the $\mathrm{Yb}$ concentration increased further. Based on Equation (6), an increase in $\varepsilon_{33}$ leads to a decrease in $k_{33}^{2}$. The value of $\varepsilon_{33}$ was generally unaffected by the addition of $\mathrm{Yb}$ up to $x=0.778$, whereas the $k_{33}^{2}$ value increased with increasing $\mathrm{Yb}$ concentration up to $x=0.778$. The $k_{33}^{2}$ values for YbAlN calculated in this work are in good agreement with the computational results by Yanagitani et al. [15] in compositions with low Yb content. The experimental $k_{33}^{2}$ values determined from thin film samples tend to be lower than the calculated results 
obtained in this study. In general, the piezoelectric properties of a thin film are affected by (i) the crystallinity of the wurtzite phase, (ii) the orientation of the crystals, and (iii) the distribution of the polarization direction. Since the computations assume a single crystal, the calculated piezoelectric properties tend to be higher than those determined on the basis of experiments. Therefore, it is expected that the calculated results are higher than the experimental values.
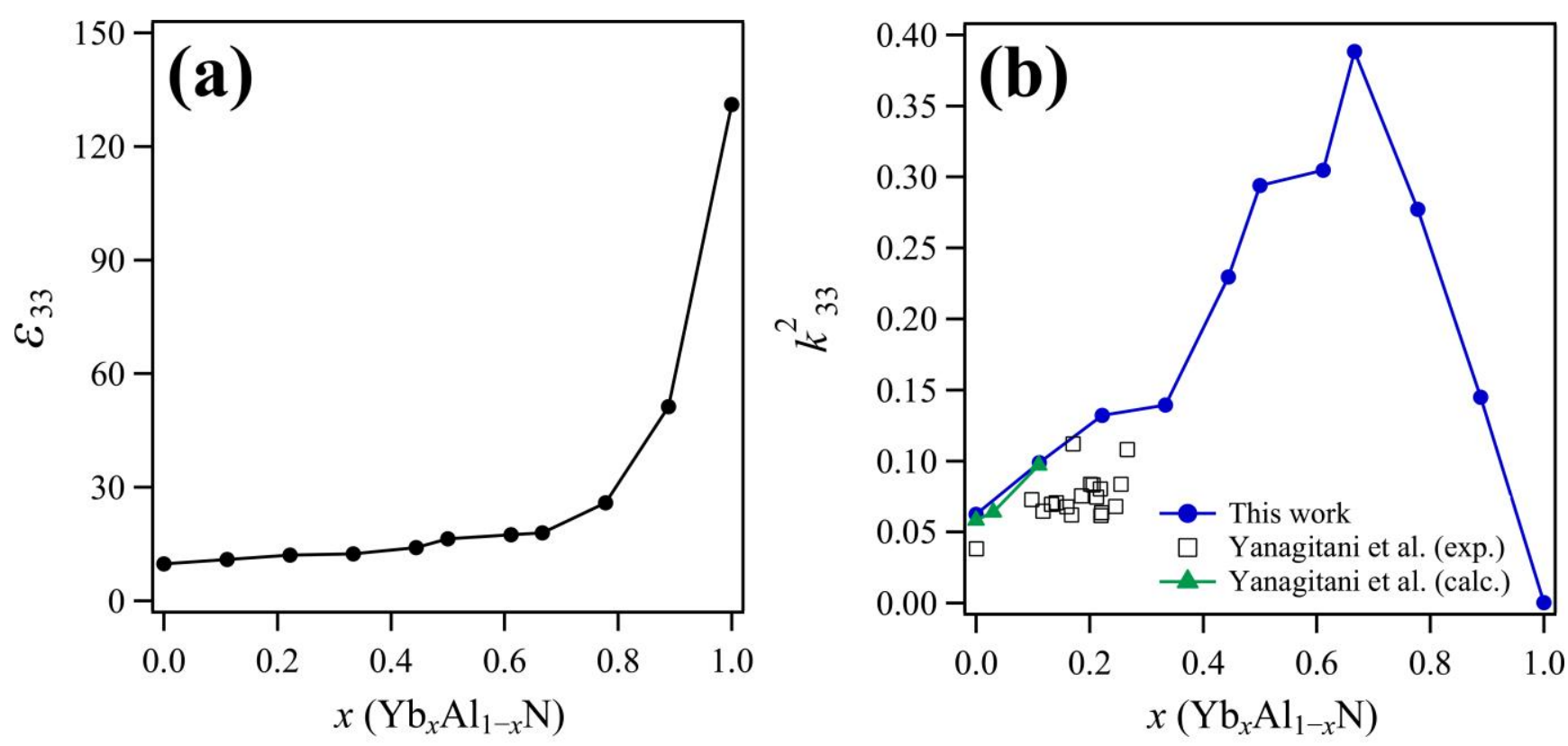

Figure 6. (a) Dielectric constant, $\varepsilon_{33}$, of $\mathrm{Yb}_{x} \mathrm{Al}_{1-x} \mathrm{~N}$ as a function of $\mathrm{Yb}$ content; and (b) electromechanical coupling coefficient, $k_{33}^{2}$, of $\mathrm{Yb}_{x} \mathrm{Al}_{1-x} \mathrm{~N}$ as a function of $\mathrm{Yb}$ content. The blue trace represents the calculated results from this work, and the black squares and green line represent the experimental and calculated results, respectively, as reported by Yanagitani et al. [15].

On the basis of the calculated $d_{33}$ and $k_{33}^{2}$ values, YbAlN is predicted to have a superior piezoelectric performance relative to AlN and to have properties comparable to ScAlN which has garnered attention as a piezoelectric material for radio frequency filters in telecommunication devices [13]. To obtain an even greater piezoelectric response with $\mathrm{YbAlN}$, higher concentrations of $\mathrm{Yb}$ should be dissolved into wurtzite AlN. Yanagitani et al. reported that the maximum solubility of $\mathrm{Yb}$ in AlN is about $x=0.25$ [15]; however, the maximum solubility of $\mathrm{Yb}$ is believed to vary depending on the preparation conditions of the thin film. In fact, the maximum solubility of Sc in AlN also varies depending on the preparation conditions and the substrate material $[35,36]$. The calculated mixing enthalpy of YbAlN (Figure 2) shows that the cross point of the wurtzite and the rock salt phases is similar to that in ScAlN $(x=0.6)[11,16]$. Since the maximum solubility of Sc in AlN is obtained at about $x=0.4$ (experimentally), the same level of solubility can be obtained for $\mathrm{YbAlN}$ by utilizing optimized preparation conditions, including the most appropriate substrate.

\section{Conclusions}

This study investigated the piezoelectric properties of $\mathrm{Yb}_{x} \mathrm{Al}_{1-x} \mathrm{~N}$ using first-principles calculations. The wurtzite phase was revealed to be more stable than rock salt phase in compositions with up to around $x=0.6$. Additionally, the piezoelectric stress constant, $e_{33}$, increased and the elastic constant, $C_{33}$, decreased with increasing $\mathrm{Yb}$ concentration (similar to the widely reported ScAlN), and as a result, the piezoelectric constant $d_{33}$ improved. The maximum calculated $d_{33}$ value was greater than $100 \mathrm{pC} / \mathrm{N}$, which is comparable to the theoretical $d_{33}$ value of ScAlN; therefore, YbAlN is also expected to be a suitable candidate for high-performance piezoelectric materials. One of the reasons for the enhancement of $d_{33}$ 
is the contribution of elastic softening due to the decreased $C_{33}$ value. The $e_{33}^{\text {nonclamped }}$ term, which is derived from the atomic displacement induced by macroscopic strain, provided the largest contribution to the enhancement of $e_{33}$. The strain sensitivity $(d u / d \varepsilon)$ of the internal parameter, $u$, exhibited the same tendency as the $\mathrm{Yb}$ concentration dependence of $e_{33}$, which indicates that it is an important factor in $e_{33}$ improvement. It was found that the dielectric constant, $\varepsilon_{33}$, generally increased with increasing $\mathrm{Yb}$ concentrations; however, the electromechanical coupling coefficient, $k_{33}^{2}$, increased only up to $x=0.778$, which was likely because of the lower values of $\varepsilon_{33}$ within this range.

Author Contributions: Conceptualization, M.U. and S.A.A.; methodology, K.H.; software, H.Y.; validation, Y.M., H.Y.; formal analysis, K.H.; investigation, K.H.; resources, H.Y.; data curation, K.H., Y.M.; writing — original draft preparation, K.H.; writing—review and editing, S.A.A.; visualization, K.H.; supervision, H.Y., M.U., M.A.; project administration, H.Y., M.A.; funding acquisition, K.H., M.A. All authors have read and agreed to the published version of the manuscript.

Funding: This research was funded by an internal grant from AIST and a Grant-in-Aid for Scientific Research from the Japan Society for the Promotion of Science (JSPS KAKENHI Grant Number 19K15302).

Institutional Review Board Statement: Not applicable.

Informed Consent Statement: Not applicable.

Data Availability Statement: Data sharing not applicable.

Acknowledgments: Some of the calculations were performed at the facilities of the Research Institute for Information Technology, Kyushu University. The authors would like to thank Y. Amano and K. Furuta at Kyushu University for their discussion.

Conflicts of Interest: The authors declare no conflict of interest.

\section{References}

1. Akiyama, M.; Kamohara, T.; Kano, K.; Teshigahara, A.; Takeuchi, Y.; Kawahara, N. Enhancement of Piezoelectric Response in Scandium Aluminum Nitride Alloy Thin Films Prepared by Dual Reactive Cosputtering. Adv. Mater. 2009, 21, 593-596. [CrossRef] [PubMed]

2. Uehara, M.; Shigemoto, H.; Fujio, Y.; Nagase, T.; Aida, Y.; Umeda, K.; Akiyama, M. Giant increase in piezoelectric coefficient of AlN by Mg-Nb simultaneous addition and multiple chemical states of Nb. Appl. Phys. Lett. 2017, 111, 112901. [CrossRef]

3. Anggraini, S.A.; Uehara, M.; Yamada, H.; Akiyama, M. Mg and Ti codoping effect on the piezoelectric response of aluminum nitride thin films. Scr. Mater. 2019, 159, 9-12. [CrossRef]

4. Tasnadi, F.; Alling, B.; Hoglund, C.; Wingqvist, G.; Birch, J.; Hultman, L.; Abrikosov, I.A. Origin of the Anomalous Piezoelectric Response in Wurtzite $\mathrm{Sc}_{\mathrm{x}} \mathrm{Al}_{1-\mathrm{x}} \mathrm{N}$ Alloys. Phys. Rev. Lett. 2010, 104, 137601. [CrossRef] [PubMed]

5. Manna, S.; Brennecka, G.L.; Stevanovic, V.; Ciobanu, C.V. Tuning the piezoelectric and mechanical properties of the AlN system via alloying with YN and BN. J. Appl. Phys. 2017, 122, 105101. [CrossRef]

6. Manna, S.; Talley, K.R.; Gorai, P.; Mangum, J.; Zakutayev, A.; Brennecka, G.L.; Stevanovic, V.; Ciobanu, C.V. Enhanced Piezoelectric Response of AlN via CrN Alloying. Phys. Rev. Appl. 2018, 9, 034026. [CrossRef]

7. Tholander, C.; Tasnadi, F.; Abrikosov, I.A.; Hultman, L.; Birch, J.; Alling, B. Large piezoelectric response of quarternary wurtzite nitride alloys and its physical origin from first principles. Phys. Rev. B 2015, 92, 174119. [CrossRef]

8. Yokoyama, T.; Iwazaki, Y.; Onda, Y.; Nishihara, T.; Sasajima, Y.; Ueda, M. Effect of Mg and Zr Co-Doping on Piezoelectric AlN Thin Films for Bulk Acoustic Wave Resonators. IEEE Trans. Ultrason. Ferroelectr. Freq. Control 2014, 61, 1322-1328. [CrossRef]

9. Iwazaki, Y.; Yokoyama, T.; Nishihara, T.; Ueda, M. Highly enhanced piezoelectric property of co-doped AlN. Appl. Phys. Express 2015, 8, 061501. [CrossRef]

10. Yokoyama, T.; Iwazaki, Y.; Nishihara, T.; Tsutsumi, J. Dopant concentration dependence of electromechanical coupling coefficients of co-doped AlN thin films for BAW devices. In Proceedings of the 2016 IEEE International Ultrasonics Symposium (IUS), Tours, France, 18-21 September 2016.

11. Talley, K.R.; Millican, S.L.; Mangum, J.; Siol, S.; Musgrave, C.B.; Gorman, B.; Holder, A.M.; Zakutayev, A.; Brennecka, G.L. Implications of heterostructural alloying for enhanced piezoelectric performance of (Al,Sc)N. Phys. Rev. Mater. 2018, 2, 063802. [CrossRef]

12. Fei, C.L.; Liu, X.L.; Zhu, B.P.; Li, D.; Yang, X.F.; Yang, Y.T.; Zhou, Q.F. AlN piezoelectric thin films for energy harvesting and acoustic devices. Nano Energy 2018, 51, 146-161. [CrossRef]

13. Liu, Y.; Cai, Y.; Zhang, Y.; Tovstopyat, A.; Liu, S.; Sun, C.L. Materials, Design, and Characteristics of Bulk Acoustic Wave Resonator: A Review. Micromachines 2020, 11, 630. [CrossRef] [PubMed] 
14. Fichtner, S.; Wolff, N.; Lofink, F.; Kienle, L.; Wagner, B. AlScN: A III-V semiconductor based ferroelectric. J. Appl. Phys. 2019, 125, 114103. [CrossRef]

15. Yanagitani, T.; Jia, J.J. ScAlN polarization inverted resonators and enhancement of $\mathrm{k}_{\mathrm{t}}^{2}$ in new YbAlN materials for BAW devices. In Proceedings of the 2019 IEEE International Ultrasonics Symposium (IUS), Glasgow, UK, 6-9 October 2019; pp. 894-899. [CrossRef]

16. Moram, M.A.; Zhang, S. ScGaN and ScAlN: Emerging nitride materials. J. Mater. Chem. A 2014, 2, 6042-6050. [CrossRef]

17. Momida, H.; Oguchi, T. Effects of lattice parameters on piezoelectric constants in wurtzite materials: A theoretical study using first-principles and statistical-learning methods. Appl. Phys. Express 2018, 11, 041201. [CrossRef]

18. Zunger, A.; Wei, S.H.; Ferreira, L.G.; Bernard, J.E. Special Quasirandom Structures. Phys. Rev. Lett. 1990, 65, 353-356. [CrossRef]

19. van de Walle, A. Multicomponent multisublattice alloys, nonconfigurational entropy and other additions to the Alloy Theoretic Automated Toolkit. Calphad J. 2009, 33, 266-278. [CrossRef]

20. Kresse, G.; Furthmuller, J. Efficiency of ab-initio total energy calculations for metals and semiconductors using a plane-wave basis set. Comput. Mater. Sci. 1996, 6, 15-50. [CrossRef]

21. Kresse, G.; Furthmuller, J. Efficient iterative schemes for ab initio total-energy calculations using a plane-wave basis set. Phys. Rev. B 1996, 54, 11169-11186. [CrossRef]

22. Perdew, J.P.; Burke, K.; Ernzerhof, M. Generalized gradient approximation made simple. Phys. Rev. Lett. 1996, 77, 3865-3868. [CrossRef]

23. Kresse, G.; Joubert, D. From ultrasoft pseudopotentials to the projector augmented-wave method. Phys. Rev. B 1999, 59, 1758-1775. [CrossRef]

24. Blochl, P.E. Projector Augmented-Wave Method. Phys. Rev. B 1994, 50, 17953-17979. [CrossRef] [PubMed]

25. Monkhorst, H.J.; Pack, J.D. Special Points for Brillouin-Zone Integrations. Phys. Rev. B 1976, 13, 5188-5192. [CrossRef]

26. Larson, P.; Lambrecht, W.R.L.; Chantis, A.; van Schilfgaarde, M. Electronic structure of rare-earth nitrides using the LSDA plus U approach: Importance of allowing $4 \mathrm{f}$ orbitals to break the cubic crystal symmetry. Phys. Rev. B 2007, 75. [CrossRef]

27. Kingsmith, R.D.; Vanderbilt, D. Theory of Polarization of Crystalline Solids. Phys. Rev. B 1993, 47, 1651-1654. [CrossRef]

28. Vanderbilt, D.; Kingsmith, R.D. Electric Polarization as a Bulk Quantity and Its Relation to Surface-Charge. Phys. Rev. B 1993, 48, 4442-4455. [CrossRef] [PubMed]

29. Resta, R. Macroscopic Polarization in Crystalline Dielectrics-The Geometric Phase Approach. Rev. Mod. Phys. 1994, 66, 899-915. [CrossRef]

30. Li, D.X.; Oyamada, A.; Hashi, K.; Haga, Y.; Matsumura, T.; Shida, H.; Suzuki, T.; Kasuya, T.; Donni, A.; Hulliger, F. Study of Physical-Properties of YB-Monopnictides. J. Magn. Magn. Mater. 1995, 140, 1169-1170. [CrossRef]

31. Watt, J.P.; Peselnick, L. Clarification of the Hashin-Shtrikman Bounds on the Effective Elastic-Moduli of Polycrystals with Hexagonal, Trigonal, and Tetragonal Symmetries. J. Appl. Phys. 1980, 51, 1525-1531. [CrossRef]

32. Wang, J.; Zhao, M.; Jin, S.F.; Li, D.D.; Yang, J.W.; Hu, W.J.; Wang, W.J. Debye temperature of wurtzite AIN determined by X-ray powder diffraction. Powder Differ. 2014, 29, 352-355. [CrossRef]

33. Jain, A.; Ong, S.P.; Hautier, G.; Chen, W.; Richards, W.D.; Dacek, S.; Cholia, S.; Gunter, D.; Skinner, D.; Ceder, G.; et al. Commentary: The Materials Project: A materials genome approach to accelerating materials innovation. APL Mater. 2013, 1, 011002. [CrossRef]

34. Petousis, I.; Mrdjenovich, D.; Ballouz, E.; Liu, M.; Winston, D.; Chen, W.; Graf, T.; Schladt, T.D.; Persson, K.A.; Prinz, F.B. High-throughput screening of inorganic compounds for the discovery of novel dielectric and optical materials. Sci. Data 2017, 4, 160134. [CrossRef] [PubMed]

35. Hoglund, C.; Birch, J.; Alling, B.; Bareno, J.; Czigany, Z.; Persson, P.O.A.; Wingqvist, G.; Zukauskaite, A.; Hultman, L. Wurtzite structure $\mathrm{Sc}_{1-\mathrm{x}} \mathrm{Al}_{\mathrm{x}} \mathrm{N}$ solid solution films grown by reactive magnetron sputter epitaxy: Structural characterization and firstprinciples calculations. J. Appl. Phys. 2010, 107, 123515. [CrossRef]

36. Hoglund, C.; Bareno, J.; Birch, J.; Alling, B.; Czigany, Z.; Hultman, L. Cubic $\mathrm{Sc}_{1-\mathrm{x}} \mathrm{Al}_{\mathrm{x}} \mathrm{N}$ solid solution thin films deposited by reactive magnetron sputter epitaxy onto ScN(111). J. Appl. Phys. 2009, 105, 113517. [CrossRef] 